\title{
Adaptación de una escala de depresión en profesores mexicanos universitarios: un estudio preliminar ${ }^{1}$
}

\author{
Adaptation of a depression scale \\ for Mexican university faculty: A preliminary study
}

\author{
Nayeli Melisa Rodríguez-Leonardo², José Horacio Tovalin Ahumada \\ y Marlene Rodríguez Martínez
}

\begin{abstract}
RESUMEN
Introducción: La docencia es un trabajo que implica dedicar gran parte del tiempo a cumplir con demandas laborales, lo que genera enfermedades físicas o trastornos emocionales tales como la depresión; tal trastorno lo padecen cerca de cuatro millones de personas en México, por lo es esencial detectar oportunamente la sintomatología depresiva. Uno de los instrumentos utilizados como método de screening es la adaptación de la Escala de Depresión en población trabajadora (ED-T), la cual ha demostrado tener propiedades psicométricas adecuadas para su uso en la misma. Objetivo: Valorar las propiedades psicométricas de la versión ED-T en una muestra de profesores universitarios de la Ciudad de México. Método: Se aplicó la escala a 206 participantes y se llevó a cabo un análisis factorial exploratorio para evaluar la pertinencia del modelo; se calculó la fiabilidad con el coeficiente Omega de McDonald (1985) y se analizó la validez concurrente de la escala por medio de la correlación de Pearson con la subescala de Afecto Negativo del PANAS y un índice de percepción general de salud. Resultados: Las pruebas de Bartlett y Kaiser-Meyer-Olkin demostraron la pertinencia de dicho análisis. La fiabilidad de la escala total fue alta y el análisis de la validez concurrente mostró una correlación positiva y significativa con la Subescala de Afecto Negativo, y negativa con el estado de salud percibido. Discusión: La adaptación de la ED-T mostró propiedades psicométricas apropiadas para profesores. Se propone una versión de trece ítems con cuatro dimensiones: "Preocupaciones e inquietudes", "Dificultad en habilidades cognoscitivas", "Disminución o ausencia de interés y energía” y "Estado de ánimo negativo".
\end{abstract}

Palabras clave: Depresión; Tests psicológicos; Escala; Psicometría; Docencia.

\begin{abstract}
Introduction: Teaching is a job that implies spending extensive time satisfying diverse demands and expectations. These conditions may foster physical illness and mental conditions, such as depressive symptoms. In Mexico, over four million people suffer this disorder and it is therefore important to promote early detection. The Depression Scale (DS) has been frequently used in workers due to its adequate psychometric properties. Objective: The present study aimed at validating the Spanish version of the Depression Scale (DS-W) in a group of university teachers in Mexico City. Method: The SD-W was applied to 206 university teachers. The factorial model was corroborated with the Bartlett's sphericity test and the Kaiser-Meyer-Olkin test. The scale's realiability was tested with the McDonald's Omega coefficient. Concurrent validity was verified with the correlation of the
\end{abstract}

\footnotetext{
${ }^{1}$ Este estudio fue financiado por Dirección General de Asuntos del Personal Académico en el Programa de Apoyo a Proyectos de Investigación e Innovación Tecnológica (PAPIIT) IG300-315.

${ }^{2}$ Carrera de Psicología, Facultad de Estudios Superiores Zaragoza, Universidad Nacional Autónoma de México, Guelatao No. 66, Col. Ejército de Oriente, Del. Iztapalapa, 09230, Ciudad de México, correo electrónico: melisa.rodriguez.leonardo@gmail.com. Artículo recibido el 15 de junio y aceptado el 8 de septiembre de 2017.

${ }^{3}$ Especialización en Salud en el Trabajo, Facultad de Estudios Superiores Zaragoza, Campus II. Batalla 5 de mayo esq. fuerte de Loreto, Col. Ejército de Oriente, Delegación Iztapalapa, 09230, Ciudad de México.
} 
DS-W with the Negative Affect Subscale and a subjective indicator of health. Results: Both tests showed consistency and pertinence of the factorial analysis. The scale's reliability was high, and the concurrent validity showed a significant positive correlation with the DS, and a negative one with low perceived health status. Discussion: This validation of the SD-T showed adequate psychometric properties. The 13-item version tested in this study revealed four dimensions: "Worries and concerns", "Difficulty in cognitive skills", "Lack of interest and energy", and "Negative mood".

Key words: Depression; Psychological tests; Scale; Psychometrics; Teaching.

\section{INTRODUCCIÓN}

工 as personas laboralmente activas enfrentan actualmente situaciones difíciles generadas por sus condiciones de trabajo o por situaciones personales estresantes que afectan su salud y desempeño profesional, por lo que es necesario contar con instrumentos de diagnóstico que sean útiles para la vigilancia adecuada y el diagnóstico y tratamiento oportunos de su estado de salud mental en contextos organizacionales (Martínez, Martínez, Esquivel y Velasco, 2007).

Es indudable que una actividad laboral intensa, prolongada y estresante, sin los periodos adecuados de descanso, constituye un campo propicio para el desarrollo de enfermedades físicas y psíquicas en el sector de los servicios, como ocurre en el caso de la docencia, actividad que implica una elevada demanda psicológica que ha llevado a muchos de estos profesionales a padecer fenómenos como los llamados "karoshi" o "burnout" (Ramírez y Mercado, 2008).

De acuerdo con el Instituto Nacional de Estadística y Geografía (INEGI) (2016), en 2014 había 1'599,727 docentes en México, de los cuales $27.8 \%$ impartía clases en el nivel superior. Hoy, este sector de la población se enfrenta a condiciones de empleo frecuentemente desfavorables, con pocas oportunidades de desarrollo y con exigencias de las organizaciones educativas que en ocasiones sobrepasan sus recursos, por lo que deben dedicar horas extras a su jornada laboral, trabajar en ambientes laborales poco saludables, con escaso apoyo social de superiores y compañeros, e incluso sin la posibilidad de adquirir las habilidades necesarias para hacer frente a las demandas. En la actualidad, las condiciones sociales y el prestigio de que antes gozaba la docencia han ido disminuyendo, lo que en muchos casos tiene como consecuencia que los docentes sean cada vez más vulnerables a sufrir diversas enfermedades de índole mental (Contreras, Veytia y Huitrón, 2009).

Dadas estas condiciones de trabajo, se han hecho diversos estudios en tales profesionales, encontrándose una elevada incidencia de patología mental, como burnout, ansiedad, estrés, depresión y fatiga crónica, así como enfermedades físicas, entre las que se hallan las cardiopatías y los trastornos digestivos (Castillo, Fernández y López, 2014; Linares y Gutiérrez, 2012; Rivero y Cruz, 2010). Además, se ha encontrado una prevalencia de depresión en comorbilidad con otros trastornos emocionales, como ansiedad y estrés, y con enfermedades físicas, como dolores musculares y cardiopatías (Ramos, 2012; Sieglin y Ramos, 2007).

La depresión es un trastorno que se caracteriza por una alteración crónica en tres aspectos principales: afectivo, cognitivo y somático. Los síntomas más frecuentes son humor deprimido, fatiga, cambios en el apetito y el sueño, pérdida de interés por actividades y pesimismo (Asociación Psiquiátrica Estadoundidense [APE], 2014; Schwartz y Schwartz, 1993).

De acuerdo con la Organización Mundial de la Salud (2016), se estima que 350 millones de personas en todo el mundo padecen depresión, por lo que se ha convertido en la causa principal de suicidio y de discapacidad asociada con padecimientos físicos. En México, las encuestas nacionales sobre salud mental indican que la depresión es uno de los trastornos afectivos más frecuentes; la Secretaría de Salud (2001) ha reportado que aproximadamente cuatro millones de personas la padecen, siendo más frecuente entre personas de escasos recursos, adultos mayores y mujeres. Pese a su alta prevalencia, no es tratada de manera adecuada.

Debido a la creciente prevalencia de la depresión y el grado de afectación individual y social que trae consigo, se ha recomendado que los médicos de primer contacto dispongan de las herramientas y los conocimientos necesarios para detectar sus síntomas de manera temprana (Calvo y Jaramillo, 2015; Vargas, Heinze, García, Fernán- 
dez y Dickinson, 2004; Vargas y Villamil, 2016; Zarragoitia, 2006), pero sin usarlos indiscriminadamente porque ello podría conducir a sobrediagnosticarla. Como apoyo a estas herramientas diagnósticas de tamizaje, hoy se cuenta con manuales y guías útiles para corroborar lo encontrado con instrumentos cortos de evaluación, lo que ofrece un panorama más completo del cuadro diagnóstico (Heinze y Camacho, 2010; Viniega, 2008).

Además de esas escalas, Brink et al. (1982) propusieron un instrumento diagnóstico de trastornos del ánimo de fácil aplicación: la Escala Geriátrica de Depresión (EGD en lo sucesivo). Hasta ese momento, los instrumentos para evaluar la depresión se diseñaban para detectar la prevalencia de síntomas físicos más que psicológicos, y dado que con el paso del tiempo los síntomas físicos pueden asociarse a condiciones propias de la edad, los autores se enfocaron en los síntomas de tipo cognitivo. Para ello, elaboraron una escala conformada por 30 ítems con un formato de respuesta dicotómica, lo que reducía la confusión al momento de responder y facilitaba su utilización como un instrumento autoaplicable. Dicha escala obtuvo un coeficiente alfa de Cronbach de .94, que indica un nivel alto de consistencia interna. La puntuación total de los ítems de la EGD permitían diferenciar entre personas deprimidas y no deprimidas, lo que hizo posible contar con un instrumento de tamizaje sensible que, al complementarlo con una entrevista clínica, apoyaba el diagnóstico y el tratamiento oportunos.

Yesavage et al. (1983) propusieron que el punto de corte fuese de +11 puntos para mejorar la especificidad y sensibilidad de la versión de 30 reactivos y, dadas las condiciones y resultados favorables que brindaba la EGD, se la utilizó principalmente en ámbitos clínicos con adultos mayores. Más tarde, los autores elaboraron una versión breve de 15 ítems, cuyos resultados fueron igualmente favorables, pues ambas versiones tuvieron una correlación alta entre sí $(\mathrm{r}=0.84 ; p<0.001)$, pudiendo así diferenciar a las personas deprimidas y no deprimidas (Yesavage y Sheikh, 1986).

En algunos países latinoamericanos se han hecho validaciones de esta última versión con resultados aceptables y una consistencia interna de .78 (Campo, Urruchurtu, Sola, Vergara y Cogollo, 2008; Martínez et al., 2005). En población mexi- cana, los análisis de consistencia interna (coeficiente alfa de .84) y de validez concurrente han comprobado que la escala puede discriminar adecuadamente entre los indicadores altos y bajos de depresión (Acosta y García, 2007; Ávila, Garant y Aguilar, 2006).

En el ámbito clínico, es importante que al evaluar la depresión se incluyan entrevistas y guías diagnósticas psiquiátricas; no obstante, en los entornos laborales es necesario contar con herramientas de tamizaje en diversos sectores u ocupaciones que permitan identificar grupos de riesgo y fomentar así la prevención y promoción de la salud mental en el centro de trabajo, tal como exige la actual Norma Mexicana sobre Factores de Riesgo Psicosocial (Secretaría de Gobernación, 2014).

Las características de la EGD, su sensibilidad y facilidad de uso hacen que se considere muy útil para el estudio de poblaciones trabajadoras en México. Recientemente fue utilizada y adecuada a la población trabajadora no geriátrica de la industria petrolera, obteniéndose una buena consistencia interna (coeficiente alfa de .85), lo que permite identificar a trabajadores con síntomas de depresión en las diferentes áreas laborales de la empresa; además, los resultados mostraron que la EGD, al tener buenos índices de confiabilidad, también es útil en muestras de personas de menor edad. De esta adecuación de la EGD se obtuvo previamente la Escala de Depresión para Trabajadores (ED-T) (Tovalin, Rodríguez, Unda y Sandoval, 2011), que fue la versión utilizada en el presente estudio en virtud de que las características demográficas de ambas muestras de trabajadores eran similares, por lo que se pretendió adaptarla para su empleo en distintos contextos laborales.

El presente es un estudio preliminar que tuvo como objetivo adaptar la escala ED-T a una muestra de docentes de nivel superior para detectar síntomas depresivos. Se muestran las propiedades psicométricas de la escala y se corrobora la validez concurrente con la Subescala de Afecto Negativo (PANAS), de Robles y Páez (2003), y el estado de salud percibido. Al constituir un primer acercamiento a esta muestra, el estudio no incluye un grupo de personas con diagnóstico clínico de depresión y uno más de comparación, por lo que no se reporta aquí la sensibilidad y especificidad del instrumento. 
Sin embargo, el análisis realizado permite mostrar la utilidad de la escala ED-T en este sector laboral en personas con un menor rango de edad para usarlo como una herramienta para identificar grupos de riesgo e intervenir en la promoción de entonos favorables en ámbitos ocupacionales (cf. Secretaría de Gobernación, 2014).

\section{MÉTODO}

\section{Muestra y sedes del estudio}

La muestra estuvo conformada por docentes de universidades públicas y privadas de la Ciudad de México y del área metropolitana, a quienes se hizo una invitación a participar en el estudio tras una previa hecha a las coordinaciones o secretarías de la institución. Participaron en total 206 docentes de nivel superior, de los cuales 56.3\% fueron mujeres, con una edad media de 47.4 años (D.E. $=10.59)$.

De estos docentes, $42.8 \%$ contaba con estudios de maestría; el rango de horas frente a grupo fue de dos a cuarenta horas semanales $(\mathrm{M}=17.9$, D.E. $=8.89$ ), y de 17.5 años en promedio (D.E. $=$ 11.05) su antigüedad en la docencia.

El mayor porcentaje de docentes pertenecía al área de Ciencias Biológicas, Químicas y de la Salud (41.8\%), y $84.2 \%$ de ellos impartía clases en licenciatura, $3.5 \%$ en especialidad y $2.5 \%$ en maestría.

\section{Instrumentos}

Se aplicó a los docentes una ficha de identificación para los datos sociodemográficos (edad, sexo y escolaridad) y laborales (jornada, alumnos atendidos y área), así como los siguientes instrumentos:

Escala de Depresión para Trabajadores (ED-T) (Adaptación por Tovalin et al. [2011] de la Escala Geriátrica de Depresión de Yesavage et al. [1983]). Consta de 30 ítems de respuesta dicotómica y es autoaplicable. Se asigna un punto por cada respuesta positiva. En los ítems 1, 5, 7, 9, 15, 19, 21, 27, 29 y 30 el puntaje se invierte. La versión utilizada en población mexicana ha demostrado niveles apropiados de consistencia interna $(\alpha=.85)$.
Subescala de Afecto Negativo (PANAS) (Robles y Páez, 2003). Es una escala que permite identificar la predisposición para actuar de forma negativa ante la vida, misma que se ha reportado como un factor personal que puede agravar la condición mental. La versión validada en español tiene una consistencia interna de .90 y consta de cinco reactivos que evalúan la presencia de los afectos negativos en las últimas semanas, con un formato de respuesta Likert que va de 1 ("Muy poco o nada") a 5 ("Extremadamente").

Índice subjetivo de salud. Se usó la pregunta “CCómo considera su estado de salud actualmente?" para evaluar la percepción subjetiva del estado de salud de los participantes, con un formato de respuesta Likert con recorrido de 1 ("Malo") a 4 ("Excelente"). Se le ha utilizado previamente con trabajadores que sufren obesidad, hipertensión y diabetes mellitus, obteniendo una menor percepción de salud en los grupos de exposición.

\section{Procedimiento}

Una vez hecha la invitación a participar en el estudio, el equipo de investigación acudió a las universidades para aplicar el instrumento. Se explicaron a los participantes los objetivos del estudio y se les presentó el formato de consentimiento informado para que lo firmaran. En compensación por su participación, se les ofreció una revisión general de su salud.

\section{Análisis estadístico}

Por ser el objetivo de este estudio adaptar la escala ED-T a docentes, se empleó para su validación el análisis factorial exploratorio para reducir los factores, estableciendo como criterio descartar los ítems que saturaran menos de .40 y que aparecieran en más de tres factores. Luego, se hizo una extracción por componentes principales con rotación factorial de los componentes con el sistema varimax (Everitt y Wykes, 2001); se realizaron las pruebas de esfericidad de Bartlett para determinar si los reactivos estaban interrelacionados (Nunnally y Bernstein, 1995), así como la medida de adecuación muestral de Kaiser-Meyer-Olkin (KMO) para analizar la matriz de intercorrelación de los datos. Para sugerir una interrelación satisfactoria 
entre los ítems se estableció un nivel de significancia igual o menor a 0.05 , y para la prueba de KMO un valor igual o superior a 0.70 .

Los factores obtenidos se sometieron al análisis de fiabilidad con el coeficiente Omega de McDonald (1985) para determinar la consistencia interna del instrumento. Distintos autores recomiendan el uso de este coeficiente de confiabilidad cuando se utilizan escalas de tipo dicotómico; el coeficiente Omega, a diferencia del alfa, trabaja con las cargas factoriales, lo que hace más estables los cálculos y refleja el verdadero nivel de fiabilidad en cuanto que no depende del número de ítems. Los valores aceptables oscilan entre .70 y .90 (Campo y Oviedo, 2008; Ventura y Caycho, 2017).

Para el análisis de la validez concurrente se empleó la correlación de Pearson, haciéndose la comparación de los puntajes obtenidos entre los profesores con y sin plaza definitiva, número de alumnos y años de antigüedad laboral para deter- minar si el instrumento tenía un comportamiento diferente en esas variables laborales.

Los datos se analizaron con el paquete estadístico SPSS, versión 23. Para el cálculo del coeficiente Omega se utilizó el programa Excel.

\section{RESULTADOS}

En el análisis factorial exploratorio se obtuvo un valor de .763 en el índice KMO, que es un valor apropiado (Everitt y Wykes, 2001), siendo asimismo significativa la prueba de esfericidad de Bartlett $(507.475, \mathrm{gl}=78, p=.000)$.

Mediante la extracción por componentes principales se eliminaron los reactivos que saturaron en menos de .40 y se adecuó la escala a 13 ítems, conformándose en cuatro componentes que explican $54.52 \%$ de la varianza muestral. La Tabla 1 muestra la saturación de los ítems en cada factor.

Tabla 1. Matriz de componentes rotados de la ED-T.

\begin{tabular}{|c|c|c|c|c|}
\hline Ítem & Factor 1 & Factor 2 & Factor 3 & Factor 4 \\
\hline ¿Está preocupado frecuentemente por el futuro? & .802 & & & \\
\hline ¿Está preocupado frecuentemente por pequeñas cosas? & .660 & & & \\
\hline ¿Está a menudo intranquilo e inquieto? & .593 & & & \\
\hline ¿Teme que le pase algo malo? & .433 & & & \\
\hline ¿Tiene esperanza en el futuro? & & .726 & & \\
\hline ¿Es difícil para usted poner en marcha nuevos proyectos? & & .619 & & \\
\hline ¿Su mente está tan clara como acostumbraba estar? & & .610 & & \\
\hline ¿Tiene problemas para concentrarse? & & .517 & & \\
\hline ¿Cree que mucha gente está mejor que usted? & & & .649 & \\
\hline ¿Ha renunciado a muchas de sus actividades e intereses? & & & .621 & \\
\hline ¿Se siente lleno de energía? & & & .616 & \\
\hline ¿Se encuentra a menudo aburrido? & & & & .793 \\
\hline ¿Se siente a menudo desanimado y melancólico? & & & & .784 \\
\hline
\end{tabular}

El factor 1 ("Preocupaciones e inquietudes") contiene los ítems 8, 11, 13 y 24 de la versión inicial del instrumento. El factor 2 ("Dificultad en habilidades cognoscitivas") incluye los ítems 5, 20, 26 y 30 , y está referido a problemas en la concentración, planificación de nuevas cosas y pesimismo respecto al futuro. En el Factor 3 ("Disminución o ausencia de interés y energía") se encuentran los ítems 2, 21 y 23, y el Factor 4 ("Estado de ánimo negativo") contiene los ítems 4 y 16.

La prueba de fiabilidad de la escala arrojó un valor de .90 en el coeficiente Omega de McDonald, lo que indica una adecuada fiabilidad interna de la escala; de acuerdo con la literatura, valores entre .70 y .90 son aceptables (Oliden y Zumbo, 2008; Ventura y Caycho, 2017). 
En el análisis de la validez concurrente, la escala resultante ED-T muestra una correlación moderada positiva del puntaje de depresión con la Subescala de Afecto Negativo $(\mathrm{r}=.570, p<.01) \mathrm{y}$ una moderada negativa con el estado de salud percibido por el individuo $(\mathrm{r}=-.327, p<.01)$. Ambos resultados ocurrieron en la dirección esperada.

De los factores laborales incluidos en la ficha de identificación, se tomó el tipo de plaza de los docentes y se comparó con los puntajes de la versión de 30 ítems y la versión propuesta de 13, encontrándose que esta última puede identificar las diferencias entre los tipos de plaza de forma similar a la versión completa $(\mathrm{t}[164]=2.34, p=0.021)$, lo que muestra que una versión breve $(\mathrm{t}[162]=2.52$, $p=0.01$ ) bien puede usarse con el fin de encontrar diferencias entre las condiciones de trabajo. Ello significa que, con la versión de 13 ítems, al comparar el puntaje de índice de depresión con el tipo de plaza se hallaron diferencias estadísticamente significativas en el puntaje total de la escala entre los docentes por asignatura $(\mathrm{M}=4$; D.E. $=3.13)$ y los que tenían plaza definitiva $(\mathrm{M}=2.84$; D.E. $=2.63)$.

Finalmente, al comparar las correlaciones entre los puntajes de las escalas y el número de alumnos atendidos y materias, se encontró que la escala de 13 ítems mostró una correlación baja pero estadísticamente significativa entre el puntaje de indicadores de depresión y el número de alumnos atendidos por semestre $(\mathrm{r}=.172, p=.05)$, así como una correlación negativa con los años de antigüedad laboral $(\mathrm{r}=-.188, p=.01)$.

\section{DISCUSIÓN}

Los resultados indican una fiabilidad adecuada en el uso de la ED-T en esta muestra de docentes mexicanos de educación superior. Mediante el análisis factorial exploratorio se obtuvieron cuatro componentes principales, los cuales coinciden con los de otros autores (Calvo y Jaramillo, 2015; Heinze y Camacho, 2010; Zarragoitia, 2006). Los síntomas tomados en cuenta resaltan un estado de ánimo negativo, preocupación por problemas en el futuro, pérdida de energía o interés por las actividades cotidianas, y problemas con aspectos cognitivos; estos síntomas también coinciden con los establecidos en el DSM-V (APE, 2014). Además, la validez concurrente tuvo buenos indicadores al relacionarse positivamente con otros de malestar emocional y negativamente con el estado percibido de salud.

Las condiciones laborales de esta muestra denotaban exceso de trabajo; los docentes reportaron tener a su cargo hasta a 500 alumnos por semestre, lo que implicaba cumplir con una gran cantidad de tareas en condiciones estresantes; por ello, en esta muestra se observó que entre mayor era el número de estudiantes, mayor fue también el puntaje obtenido en la ED-T. Dado lo anterior, se deben gestionar cambios en las condiciones laborales para brindar una mejor calidad en la educación sin sacrificar el estado de salud de los docentes.

El uso de la ED-T permitirá detectar oportunamente a quienes manifiesten sintomatología al reducirse de 30 a 13 los ítems, haciendo así más rápida su aplicación. Además, en este estudio las propiedades psicométricas de la escala mostraron validez interna y externa en una muestra con una edad media menor a la de la población a la que estaba originalmente dirigida.

La validez concurrente obtenida aquí indica que posee las cualidades psicométricas adecuadas para identificar sintomatología depresiva; sin embargo, es importante señalar que el uso de este instrumento es solamente un apoyo para el tamizaje y la canalización en caso de ser necesarios, y que se debe ampliar su estudio para determinar con precisión su sensibilidad, especificidad y punto de corte, al igual que llevar a cabo un análisis confirmatorio en este grupo de profesionales.

\section{REFERENCIAS}

Acosta Q., C.O. y García F., R. (2007). Ansiedad y depresión en adultos mayores. Psicología y Salud, 17(2), $291-300$.

Asociación Psiquiátrica Estadounidense (2014). Manual diagnóstico y estadístico de los trastornos mentales-V. México: Editorial Médica Panamericana. 
Ávila F., J.A., Garant M., P. y Aguilar N., S. (2006). Relación entre los factores que determinan los síntomas depresivos y los hábitos alimentarios en adultos mayores de México. Revista Panamericana de Salud Pública, 19(5), 321-30. doi: http:// dx.doi.org/10.1590/S1020-49892006000500005.

Brink, T.L., Yesavage, J.A., Lum, O., Heersema, P., Adey, M. y Rose, T. (1982). Screening tests for geriatric depression. Clinical Gerontology, 1(1), 37-43. doi: http://dx.doi.org/10.1300/J018v01n01_06.

Calvo G., J.M. y Jaramillo G., L.E. (2015). Detección del trastorno depresivo mayor en atención primaria. Una revisión. Revista de la Facultad de Medicina, 63(3), 471-482.

Campo A., A., Urruchurtu M., Y., Solano, T., Vergara A., J. y Cogollo, Z. (2008). Consistencia interna, estructura factorial y confiabilidad del constructo de la Escala de Yesavage para depresión geriátrica (GDS-15) en Cartagena, Colombia. Salud Uninorte, 24(1), 1-9.

Campo A., A. y Oviedo H., C. (2008). Propiedades psicométricas de una escala: la consistencia interna. Revista de Salud Pública, 10(5), 831-839. doi: http://dx.doi.org/10.1590/S0124-00642008000500015.

Castillo M., A., Fernández P., R. y López J., P. (2014). Prevalencia de ansiedad y depresión en docentes. Enfermería del Trabajo, $4,55-62$.

Contreras G., J., Veytia L., M. y Huitrón B., G. (2009). Detección del trastorno depresivo en docentes universitarios. Psicología y Salud, 19(1), 133-139.

Everitt, B.S. y Wykes, T. (2001). Diccionario de estadística para psicólogos. Madrid: Ariel.

Heinze M., G. y Camacho S., P. (2010). Guía clínica para el manejo de la depresión. México: Instituto Nacional de Psiquiatría Ramón de la Fuente Muñiz. Recuperado de http://www.inprf-cd.gob.mx/guiasclinicas/manejo_depresion.pdf.

Instituto Nacional de Estadística y Geografía (2016). Estadísticas a propósito del Día del Maestro (15 de mayo). Datos nacionales. México: INEGI. Recuperado de http:/www.inegi.org.mx/saladeprensa/aproposito/2015/maestro0.pdf.

Linares O., L. y Gutiérrez R., E. (2012). Efectos moduladores del apoyo social ante la reacción de estrés y ansiedad en profesores. Psicología y Salud, 22(1), 107-114.

Martínez de la Iglesia, J., Onís V., R., Dueñas, C., Aguado, C., Albert C., C. y Arias M., C. (2005). Abreviar lo breve. Aproximación a versiones ultracortas del cuestionario de Yesavage para el cribado de la depresión. Atención Primaria, 35(1), 14-21. doi: http://dx.doi.org/10.1157/13071040.

Martínez M., J.A., Martínez O., V.A., Esquivel M., C.G. y Velasco R., V.M. (2007). Prevalencia de depresión y factores de riesgo en el adulto mayor hospitalizado. Revista Médica del Instituto Mexicano del Seguro Social, 45(1), 21-28.

McDonald, R.P. (1985). Factor analysis and related methods. Hillsdale, NJ: Erlbaum.

Nunnally, J.C. y Bernstein, I.J. (1995). Teoría psicométrica (3 $3^{\mathrm{a}}$ ed.). México: McGraw-Hill Latinomericana.

Oliden, P.E. y Zumbo, D.B. (2008). Coeficientes de fiabilidad para escalas de respuesta categórica ordenada. Psicothema, 20(4), 896-901.

Organización Mundial de la Salud (2016). La depresión. Recuperado de: http://www.who.int/mediacentre/factsheets/fs369/es/.

Ramírez, M. y Mercado, A. (2008). El síndrome del desgaste profesional en académicos. Memorias del X Congreso Nacional de Investigación Educativa. Veracruz, Ver., 21-25 de septiembre. Recuperado de http://www.uv.es/unipsico/pdf/CESQT/Externos/2009_Mercado_y_Ramirez.pdf.

Ramos T., M.E. (2012). Conflicto familia-trabajo-familia y salud psicosomática entre académicos y académicas mexicanos. Voces del Noreste. Aportes regionales a los estudios de género. Monterrey (México): Universidad Autónoma de Nuevo León. Recuperado de https://www.researchgate.net/profile/Maria_Ramos/publication/235435499_Voces_del_Noreste_Aportaciones_a_los_estudios_de_genero/links/09e41511a4f41110e-6000000.pdf\#page=277.

Rivero R., L.F. y Cruz M., A.C. (2010). Trastornos psíquicos y psicosomáticos: problemas actuales de salud de los docentes mexicanos. Psicología y Salud, 20(2), 239-249.

Robles, R. y Páez, F. (2003). Estudio sobre la traducción al español y las propiedades psicométricas de las escalas de afecto positivo y negativo (PANAS). Salud Mental, 26(1), 69-75.

Schwartz, A, y Schwartz, R.M. (1993). Depression. Theories and treatments. Psychological, biological, and social perspectives. New York, NY: Columbia University Press.

Secretaría de Gobernación (2014). Reglamento de Seguridad y Salud en el Trabajo. Diario Oficial de la Federación, 13 de noviembre. Recuperado de http://www.dof.gob.mx/nota_detalle.php?codigo=5368114yfecha=13/11/2014.

Secretaría de Salud (2001). Programa de Acción en Salud Mental. México: Secretaría de Salud. Recuperado de http://www.salud. gob.mx/unidades/cdi/documentos/pasm_intro.pdf.

Sieglin, V. y Ramos T., M.E. (2007). Estrés laboral y depresión entre maestros del área metropolitana de Monterrey. Revista Mexicana de Sociología, 69(3), 517-551.

Tovalin, J.H., Rodríguez, M., Unda, S.G. y Sandoval, J.I. (2011). Adecuación de la Escala de Depresión de Yesavage, versión completa (30). México: UNAM.

Vargas B., E. y Villamil S., V. (2016). La salud mental y la atención primaria en México. Experiencias de un modelo de atención colaborativa. Salud Mental, 39(1), 3-9. 
Vargas B., E., Heinze, G., García, J., Fernández, M.A. y Dickinsson, M.E. (2004). La capacitación del médico general del primer nivel de atención en el manejo de la depresión. Revista de la Facultad de Medicina, 47(5), 187-191.

Ventura L., J.L. y Caycho R., T. (2017). El coeficiente Omega: un método alternativo para la estimación de la confiabilidad. Revista Latinoamericana de Ciencias Sociales, Niñez y Juventud, 15(1), 625-627.

Viniega O., A. (2008). Diagnóstico y tratamiento de la depresión en el adulto mayor en el primer nivel de atención. Evidencias y recomendaciones. México: Secretaría de Salud. Recuperado de http://www.isssteags.gob.mx/guias_praticas_medicas/gpe/ docs/IMSS-194-10-ER.pdf.

Yesavage, J.A. y Sheikh, J.I. (1986). 9/Geriatric Depression Scale (GDS). Clinical Gerontology, 5(1-2), 165-173. doi: http:// dx.doi.org/10.1300/J018v05n01_09.

Yesavage, J.A., Brink, T.L., Rose, T.L., Lum, O., Huang, V., Adey, M. y Von, O.L. (1983). Development and validation of a geriatric depression screening scale: a preliminary report. Journal of Psychiatric Research, 7(1), 37-49. doi: http://dx.doi. org/10.1016/0022-3956(82)90033-4.

Zarragoitia A., I. (2006). La depresión en atención primaria de salud mental. Revista del Hospital Psiquiátrico de La Habana, 3(2). Recuperado de http://www.revistahph.sld.cu/hph0206/hph02606.htm. 